\title{
Esofagitis necrotizante aguda en paciente inestable
}

\section{Acute necrotizing esophagitis in an unstable patient}

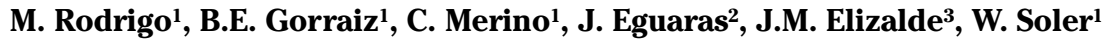

\section{RESUMEN}

La esofagitis necrotizante aguda o esófago negro es una afección rara, conocida en la literatura médica desde 1990. Aunque su mecanismo no es del todo conocido, el compromiso isquémico parece ser el factor fisiopatológico fundamental. Cuando la esofagitis necrotizante aguda es un hallazgo en el estudio endoscópico de hemorragia digestiva alta, sin desestabilización hemodinámica, el pronóstico es bueno. Presentamos un caso en el que el diagnóstico de esófago negro fue un mero hallazgo endoscópico, secundario a una situación de inestabilidad hemodinámica subyacente, con resultado fatal.

Palabras clave. Esofagitis necrotizante aguda. Rotura de aneurisma aórtico abdominal.

\begin{abstract}
Acute necrotizing esophagitis or black esophagus is a rare affection, described in the medical literature since 1990. Although its mechanism is not fully understood, ischemic compromise appears to be the fundamental physiopathological factor. When acute necrotizing esophagitis is found in the endoscopic study of an upper digestive haemorrhage, the prognosis is good. We present a case in which diagnosis of black esophagus was an endoscopic finding, secondary to a situation of underlying hemodynamic instability, with a fatal outcome.
\end{abstract}

Key words. Acute necrotizing esophagitis. Rupture of abdominal aortic aneurysm.
1. Servicio de Urgencias. Hospital Virgen del Camino. Pamplona.

2. Servicio de Aparato Digestivo. Hospital Virgen del Camino. Pamplona.

3. Servicio de Anatomía Patológica. Hospital Virgen del Camino. Pamplona.

Recepción el 6 de diciembre de 2008 Aceptación provisional el 15 de diciembre de 2008

Aceptación definitiva el 21 de enero de 2009

\section{Correspondencia:}

Marino Rodrigo Bañuelos

Servicio de Urgencias

Hospital Virgen del Camino

Irunlarrea, 4

31008 Pamplona

Tfno. 848429462

E-mail: mrb300@gmail.com 


\section{INTRODUCCIÓN}

La esofagitis necrotizante aguda (ENA) o esófago negro es una afección rara conocida en la literatura desde $1990^{1}$. Aunque su mecanismo no es del todo conocido, el compromiso isquémico parece ser el factor fisiopatológico fundamental. El síntoma más común es hematemesis o melenas, siendo una entidad a considerar en el diagnóstico diferencial de la hemorragia gastrointestinal, en particular en pacientes ancianos. Su diagnóstico de sospecha es endoscópico y el de confirmación, anatomopatológico. Ante su presencia, es necesario investigar una posible situación de hipovolemia subyacente, como ilustra el caso que presentamos a continuación.

\section{CASO CLÍNICO}

Hombre de 77 años derivado al servicio de Urgencias hospitalario desde su centro de salud para valoración de hematemesis. Como antecedentes personales el paciente refería sangrado digestivo alto dos años antes, en contexto de toma de aspirinas por proceso catarral, así como crisis renoureterales (CRU) de repetición y asma. No se disponía en ese momento de más información relativa a sus antecedentes personales. Se encontraba tomando tratamiento inhalatorio antiinflamatorio-broncodilatador; por voluntad propia el paciente había suspendido recientemente el tratamiento habitual con omeprazol. Diagnosticado tres días antes de CRU en su centro de salud por dolor lumbar bajo de perfil cólico irradiado a cuadrante inferior izquierdo (CII), había recibido diclofenaco intramuscular y aspirinas a demanda, con alivio parcial. Ante la presentación de hematemesis, se deriva para valoración.

En el servicio de Urgencias el paciente refiere, además de dicho dolor lumbar irradiado, que reconoce como de características similares a CRU

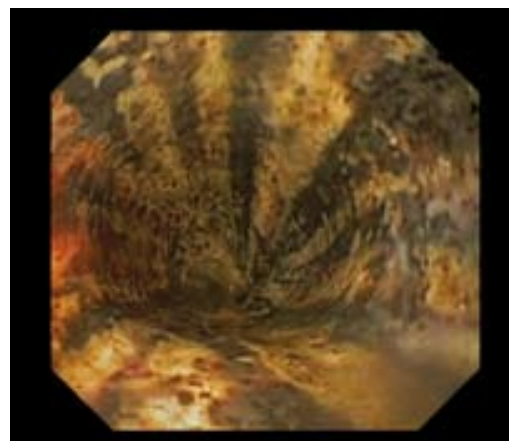

Figura 1. Mucosa esofágica de aspecto necrótico y restos de sangre. previas, la presentación en las últimas ocho horas de ardor de estómago, así como temblor al levantarse de la cama e inestabilidad, que ha condicionado caída y traumatismo nasal. Asimismo, había presentado en este período tres vómitos de contenido sanguinolento. En la exploración física destacaban buen estado general, TA de 70/50 mm de $\mathrm{Hg}$ con relleno capilar normal, FC de $90 \mathrm{lpm}, \mathrm{t}^{\mathrm{a}}$ de $36^{\mathrm{o}}$ $\mathrm{C}$; nauseoso, con palidez concomitante a las náuseas, pero basalmente normocoloreado; herida en puente nasal con restos de sangrado en fosas nasales y restos en "posos de café" en comisuras labiales; abdomen doloroso a la palpación profunda en CII, sin signos de irritación peritoneal ni aparentes masas y tacto rectal negativo para sangre y melenas. Se extrajeron muestras para analítica y pruebas cruzadas y se inició sueroterapia por dos vías venosas periféricas, que consiguieron remontar las cifras tensionales sin normalizarlas. En la analítica, Hb, 12,9 g/dL, Hto. 36,1\%, leucocitos $27.500 / \mu \mathrm{L}$ con $87 \%$ de neutrófilos, plaquetas y coagulación normales; urea $52 \mathrm{mg} / \mathrm{dL}$, creatinina 2,86 $\mathrm{g} / \mathrm{dL}$; resto normal. Ante esta situación de inestabilidad hemodinámica y hematemesis en paciente con antecedentes de hemorragia digestiva alta y tratamiento actual con fármacos potencialmente gastrolesivos, se solicitó endoscopia urgente.

La endoscopia inmediata (Figs. 1 y 2) objetivó esófago con mucosa de aspecto claramente isquémico desde los $30 \mathrm{~cm}$ y necrótico hasta los $38 \mathrm{~cm}$, así como fundus, cuerpo gástrico, incisura y antro normales, con algún resto hemático mínimo. Durante la realización de la endoscopia el paciente entró en parada cardiorrespiratoria por actividad eléctrica sin pulso. Las maniobras de resucitación resultaron infructuosas y el paciente falleció. La necropsia reveló esofagitis aguda necrotizante erosiva en los $14 \mathrm{~cm}$ distales y arteriosclerosis aórtica, predominantemente abdominal y en bifurcación de ilíacas, con aneurisma sacular en aorta abdominal de $13 \mathrm{~cm}$ de longitud y $8 \mathrm{~cm}$ de diámetro roto, hemorragia retroperitoneal masiva de $3.000 \mathrm{cc}$, aproximadamente, y extensión a cavidad peritoneal.

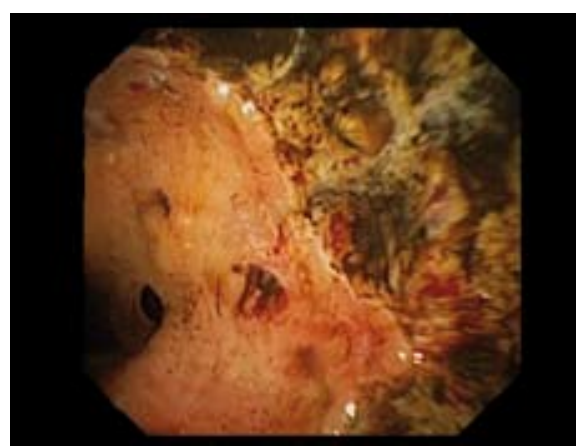

Figura 2. Mucosa esofágica necrótica y restos de sangre. 


\section{DISCUSIÓN}

La esofagitis necrotizante aguda (ENA) es una afección rara conocida en la literatura desde $1990^{1}$. Su incidencia se desconoce, pero se sospecha infradiagnosticada ${ }^{2}$. Hasta 2006 se hallaron informes referidos a solo 88 pacientes, 70 hombres y $16 \mathrm{mu}-$ jeres con una edad media de 67 años $^{3}$. Se diagnosticó ENA en el $0,28 \%$ de 10.295 endoscopias realizadas por cualquier motivo durante un período de 5 años $^{4}$, así como en el $6 \%$ de endoscopias realizadas por hemorragia digestiva ${ }^{5}$.

Aunque su mecanismo no es del todo conocido, el compromiso isquémico parece ser un factor fisiopatológico fundamental. Otros factores sospechados son reflujo gastroesofágico masivo e infección esofágica. La parte afectada suelen ser los dos tercios distales del esófago, que aparecen en la endoscopia con una decoloración negra circunferencial de toda la mucosa. Por ello, esta afección recibe también el nombre de esófago negro.

Desde el punto de vista anatomopatológico, la ENA se caracteriza como una lesión mucosa aguda (Fig. 3). Al examen microscópico, la mucosa está necrótica y limitada por un infiltrado leucocitario en la submucosa alta, consistente en neutrófilos y macrófagos, con ausencia de depósitos de hemosiderina (Fig. 4). El diagnóstico de esófago negro es histológico y precisa la exclusión de otras causas, como ingestión de cáusticos.

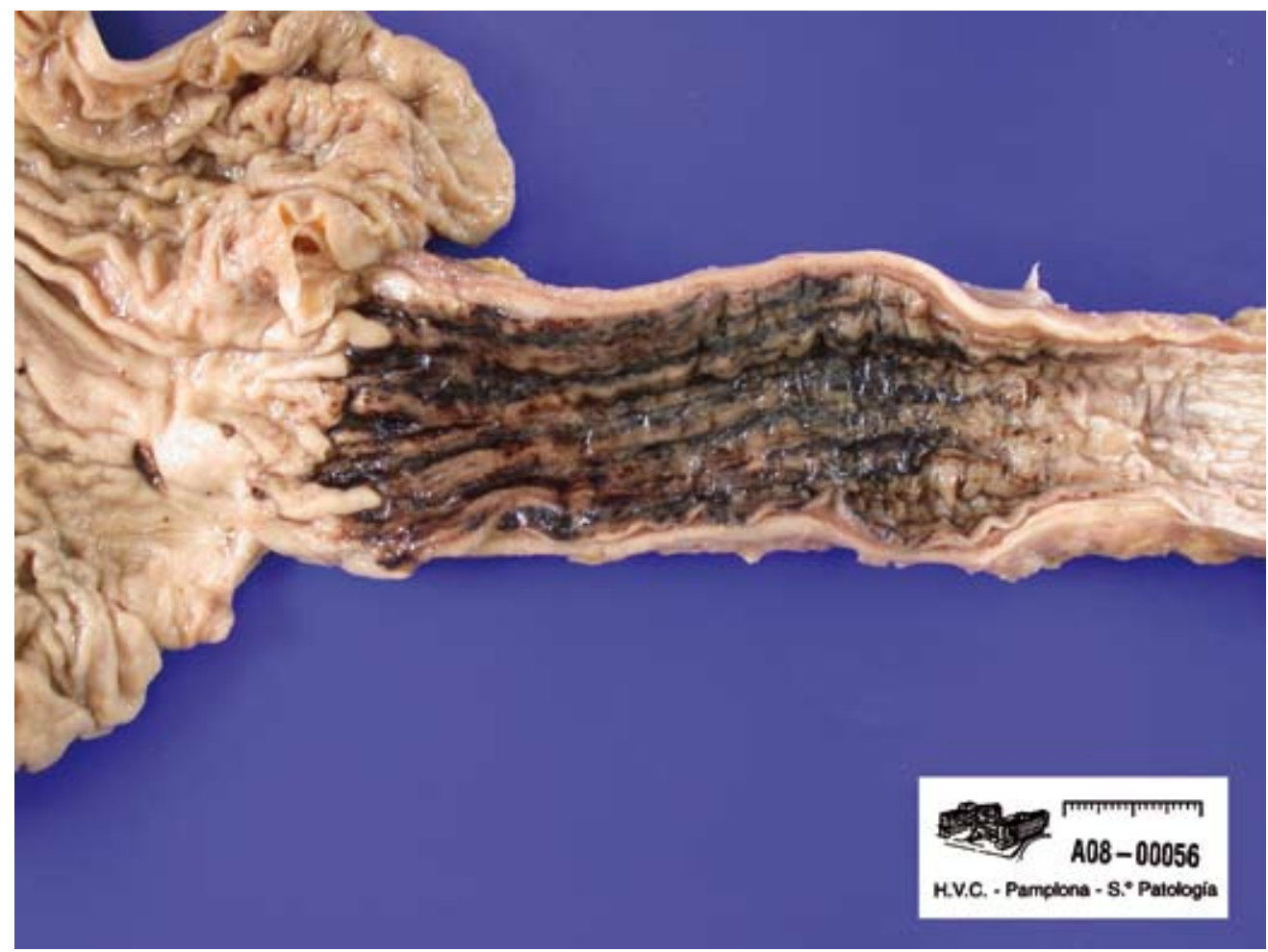

Figura 3. Pieza de necropsia.

El síntoma más común (78\%) es hematemesis o melenas ${ }^{6}$. La ENA es una entidad a considerar en el diagnóstico diferencial de la hemorragia gastrointestinal, en particular en pacientes ancianos. Complicaciones posibles incluyen estenosis, mediastinitis/ abscesos y perforación. La mortalidad global se sitúa en torno al $31,8 \%$, y su pronóstico depende más de la avanzada edad del paciente y de la comorbilidad asociada que 
de la evolución de las lesiones esofágicas. Cuando la ENA es un hallazgo en el estudio endoscópico de hemorragia digestiva alta (HDA), sin desestabilización hemodinámica, el pronóstico es bueno.

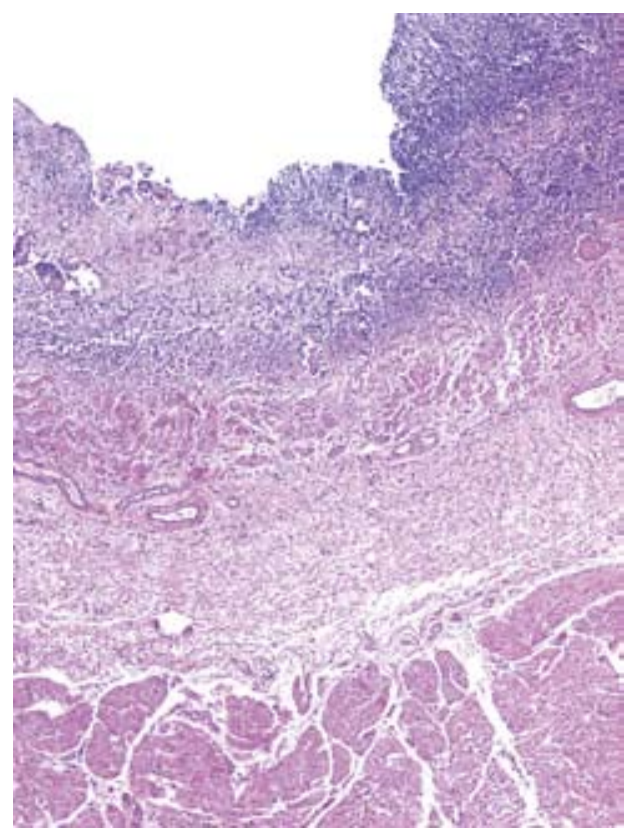

Figura 4. Aspecto macroscópico.

Un aneurisma aórtico abdominal (AAA) intacto puede producir dolor abdominal, lumbar o en flanco, que tiene un comienzo gradual y es de características vagas. El dolor agudo o intenso sugiere complicación aguda, como rotura o disección ${ }^{7}$. Se considera a la rotura de aneurisma aórtico abdominal (RAAA) otra de las "grandes imitadoras", por simular diversas patologías lumbares y abdómino-pélvicas, entre ellas la CRU. La RAAA suele producirse hacia el retroperitoneo. El 10-30\% de los pacientes tienen rotura libre intraperitoneal, que suele ser rápidamente mortal ${ }^{8}$.

En el caso presentado, la confluencia de información clínica desde los antecedentes personales, la anamnesis y la exploración física condicionó el énfasis del estudio in- mediato en la hematemesis (hemorragia digestiva alta como causa posible de la inestabilidad hemodinámica que presentaba el paciente), en lugar de en el dolor lumbar irradiado. Este se interpretó inicialmente como CRU y retrospectivamente como probable complicación de AAA. No constaba el diagnóstico de AAA en sus AP. El esófago negro fue un hallazgo endoscópico casual, resultado probable de isquemia aguda debida a hipovolemia, secundaria a su vez a sangrado aórtico abdominal, con evolución fulminante y resultado fatal. Respecto a la hematemesis, no fue la causa de la inestabilidad sino un mero epifenómeno, probablemente debido a sangre deglutida de origen nasal (epistaxis traumática) y, quizá, a sangrado leve debido a la ENA.

\section{BIBLIOGRAFÍA}

1. Goldenberg S, Wain S, Marignani P. Acute necrotizing esophagitis. Gastroenterol 1990; 2 : 493-496.

2. Ben Soussan E, Savoye G, Hochain P, Hervé S, Antonietti M, Lemoine F et al. Acute esophageal necrosis: a 1-year prospective study. Gastrointest Endosc 2002: 56: 213-217.

3. Gurvits GE, Shapsis A, Lau N, Gualtieri N, RobiLOTTI JG. Acute esophageal necrosis: a rare syndrome. J Gastroenterol 2007; 42: 29-38.

4. Augusto F, Fernandes V, Cremers VI, Oliveira AP, Lobato C, Alves AL et al. Acute necrotizing esophagitis: a large retrospective case series. Endoscopy 2004; 36: 411-415.

5. Yasuda H, Yamada M, Endo Y, Inoue K, Yoshiba M. Acute necrotizing esophagitis: role of nonsteroidal anti-inflamatory drugs. J Gastroenterol 2006; 41: 193-197.

6. Grudell AB, Mueller PS, Viggiano TR. Black esophagus: report of six cases and review of the literatura, 1963-2003. Dis Esophagus 2006; 19: 105-110.

7. JoHnson GA. Aneurismas y disección aórticas. En: Tintinalli JE, editor. Medicina de Urgencias. $5^{\text {a }}$ edición. México, McGraw-Hill Interamericana editores 2002: 468-472.

8. Bessen HA. Aneurisma de la aorta abdominal. En: Marx-Hockberger-Walls, editores. Rosen Medicina de Urgencias. Conceptos y práctica clínica. $5^{\mathrm{a}}$ edición. Madrid. Elsevier España, SA. 2003. 1176-1186. 